\title{
COMPARISON OF SURGICAL METHODS IN SACROCOCCYGEAL PILONIDAL SINUS TREATMENT
}

\author{
Hasan Cantay ${ }^{1}$, Yavuz Dasdemir ${ }^{2}$ \\ ${ }^{1}$ Kafkas University, School of Medicine, Department of General Surgery, Kars, Turkey \\ ${ }^{2}$ Kars Harakani Public Hospital, General Surgery Clinic, Kars, Turkey
}

Address for Correspondence: Hasan Cantay, Asst. Prof. Dr., E-mail: hasan_cantay@hotmail.com Received: 28.05.2021; Accepted: 04.08.2021; Available Online Date: 20.09.2021

@C Copyright 2021 by Dokuz Eylül University, Institute of Health Sciences - Available online at https://dergipark.org.tr/en/pub/jbachs

Cite this article as: Cantay H, Dasdemir Y. Comparison of Surgical Methods in Sacrococcygeal Pilonidal Sinus Treatment. J Basic Clin Health Sci 2021; 3: 126- 131.

\begin{abstract}
Purpose: Although many conservative and surgical methods have been described in the treatment of sacrococcygeal pilonidal sinus, which is a common disease of the sacrococcygeal region, affecting especially young men, recurrence rates are still high and the search for ideal treatment continues. The aim of the study is to compare the primary closure, Limberg and Karydakis flap methods for pilonidal sinus surgery in terms of wound healing, complications and recurrence and to present the results accordingly.
\end{abstract}

Methods: The data of 184 patients who were operated between 2018 and 2020 were retrospectively evaluated. Patient data on age, gender, comorbidity, Body Mass Index (BMI), smoking, recurrence, history of preoperative abscess drainage, surgical methods, length of hospital stay, postoperative drain, recurrence and complications were evaluated.

Results: No statistically significant difference was found between the treatment methods in terms of age, gender, Body Mass Index, and smoking ( $p>0.005)$. A statistically significant difference was found between the treatment methods in terms of reoperation for recurrence and preoperative abscess drainage $(p=$ $0.012)(p=0.044)$. However, a significant difference was found between the treatment methods in terms of length of hospital stay, presence of drainage, recurrence and complications $(p<0.005)$.

Conclusion: Although primary suturation has a short hospital stay, the rate of recurrence and complications is higher than the Limberg and Karydakis flap methods. In this context, it can be maintained that the Limberg and Karydakis flap methods are safer methods than the primary closure method.

Keywords: Pilonidal sinus, Recurrence, Limberg flap, Karydakis flap, Primary closure, Surgical Flaps

\section{INTRODUCTION}

Pilonidal sinus (PS) is a common disease of the sacrococcygeal region, affecting especially young men. Its incidence varies regionally and racially. Its etiology and pathogenesis have been discussed for years. It is important to treat with symptoms that are frequently recurrent, painful and affect the patient's quality of life $(1,2)$. Although many conservative and surgical methods have been defined as a treatment method, recurrence rates are still high and the search for ideal treatment continues (3-5). Although the surgical treatment to be applied by excision of the diseased area is generally accepted, the method to close the defect is controversial. Conducted within a secondary-care hospital, the present study is intended to compare the primary closure, Limberg and Karydakis flap methods for PS surgery in patients diagnosed with sacrococcygeal pilonidal sinus in terms of wound healing, complications and recurrence, and to present the results accordingly. 
Table 1: Effects of independent variables on treatment methods

\begin{tabular}{|c|c|c|c|c|c|c|c|}
\hline & & & tment Metl & & & & \\
\hline Independent & ariables & $\begin{array}{c}\text { Primary } \\
\text { closure }\end{array}$ & $\begin{array}{c}\text { Limberg } \\
\text { Flap }\end{array}$ & $\begin{array}{l}\text { Karydakis } \\
\text { Flap }\end{array}$ & Total & $\begin{array}{c}X^{2} \\
\text { value }\end{array}$ & $\begin{array}{c}P \\
\text { value }\end{array}$ \\
\hline $1 ;$ & $<26$ & $38(44,2)$ & $29(33,7)$ & $19(22,1)$ & $86(46,7)$ & 5015 & $0 \Omega 0 \% 0$ \\
\hline Age & $\geq 26$ & $45(45,9)$ & $39(39,8)$ & $14(14,3)$ & $98(53,3)$ & $2,04 J$ & 0,500 \\
\hline Condro & Female & $16(57,1)$ & $9(32,1)$ & $3(10,7)$ & $28(15,2)$ & 1202 & 0200 \\
\hline & Male & $67(42,9)$ & $59(37,8)$ & $30(19,2)$ & $156(84,8)$ & & \\
\hline DMU & $<30$ & $75(44,6)$ & $62(36,9)$ & $31(18,5)$ & $168(91,3)$ & 0000 & 0009 \\
\hline & $\geq \mathbf{3 0}$ & $8(50,0)$ & $6(37,5)$ & $2(12,5)$ & $16(8,7)$ & & \\
\hline S & Yes & $57(44,2)$ & $49(38,0)$ & $23(17,8)$ & $129(70,1)$ & 0000 & 0001 \\
\hline & No & $26(47,3)$ & $19(34,5)$ & $10(18,2)$ & $55(29,9)$ & & \\
\hline Previous & Yes & $3(16,7)$ & $8(44,4)$ & $7(38,9)$ & $18(9,8)$ & 8765 & 0010 \\
\hline Pilonidal Surgery & No & $80(48,2)$ & $60(36,1)$ & $26(15,7)$ & $166(90,2)$ & $8, / 05$ & 0,012 \\
\hline Preoperative & Yes & $4(23,5)$ & $11(64,7)$ & $2(11,8)$ & $17(9,2)$ & 6024 & 0040 \\
\hline Abscess Drainage & No & $79(47,3)$ & $57(34,1)$ & $31(18,6)$ & $167(90,8)$ & $0,2 J 4$ & $0,0+4$ \\
\hline Total & & $83(45,1)$ & $68(37,0)$ & $33(17,9)$ & $184(100,0)$ & & \\
\hline
\end{tabular}

*BMI: Body Mass Index

\section{MATERIAL AND METHOD}

In our study, the data of 184 patients who were operated with the diagnosis of sacrococcygeal pilonidal sinus in the General Surgery Clinic of XXX Hospital between January 2018 and December 2020 were retrospectively evaluated. Patient data on age, gender, comorbidity, Body Mass Index (BMI), smoking, recurrence, history of preoperative abscess drainage, surgical methods, length of hospital stay, postoperative drain, recurrence and complications were evaluated.

Independent variables of the study were age, gender, comorbidity, BMI, smoking, state of recurrence, history of preoperative abscess drainage, surgical methods performed.

Dependent variables of the study were surgical methods, length of hospital stay, presence of postoperative drain, recurrence and complication status

Three different surgical techniques were applied to the patients.

Treatment methods were as follows:

1. Primary suturing applied after cyst excision

2. Rhomboid excision applied Limberg Flap

3. Karydakis Flap applied after excision

The sample size was not selected as all operated pilonidal sinus cases between 2018-2020 were included in the study. Preoperative and postoperative antibiotherapy was applied to all patients. Approval for the study was obtained from the Ethics Committee of the XXX, with the decision no: 80576354-05099/349 and date: 09.03.2021 and written informed consent was obtained from all patients.

\section{Analysis of research data}

SPSS version 20 for Windows software package program was used in the analysis of the data. The descriptive criteria in the study are frequency and percentages, while mean value was used out of the criteria of central tendency. In addition, chi-square (Pearson Chi-Square) analyzes were used in the analysis of the data determined by counting.

\section{RESULTS}

The mean age of the patients in the study was 26.47 \pm 6.10 , and the median was 26 (16-44). When evaluated in terms of age, $53.3 \%$ of the patients are above the age of $26,46.7 \%$ are under the age of 26 . When compared in terms of age, no statistically significant difference was found between the treatment methods $(p=0.360)$. In the study, $84.8 \%$ of the patients were male; and whencompared in terms of gender, no statistically significant difference was found between the treatment methods ( $p=0.328$ ). However, it was observed that $91.3 \%$ of the patients 
Table 2: The effects of operation methods on duration of hospitalization, presence of drain, recurrence and complications

\begin{tabular}{|c|c|c|c|c|c|c|c|c|c|}
\hline \multirow{3}{*}{$\begin{array}{l}\text { Treatment } \\
\text { Methods }\end{array}$} & \multicolumn{2}{|c|}{$\begin{array}{c}\text { Duration of } \\
\text { Hospitalization }\end{array}$} & \multicolumn{2}{|c|}{ Presence of Drain } & \multicolumn{2}{|c|}{ Recurrence } & \multicolumn{2}{|c|}{ Complication } & \multirow{2}{*}{ Total $* *$} \\
\hline & $\leq 2$ days & $\geq 3$ days & Yes & No & Yes & No & Yes & No & \\
\hline & n $(\%) *$ & n $(\%)^{*}$ & n $(\%) *$ & n $(\%) *$ & n $(\%) *$ & n $(\%) *$ & n $(\%) *$ & n $(\%)^{*}$ & n (\%) \\
\hline $\begin{array}{l}\text { Primary } \\
\text { closure }\end{array}$ & $75(90,4)$ & $8(9,6)$ & $6(7,2)$ & $77(92,8)$ & $15(18,1)$ & $68(81,9)$ & $19(22,9)$ & $64(77,1)$ & $83(45,1)$ \\
\hline $\begin{array}{l}\text { Limberg } \\
\text { Flap }\end{array}$ & $52(76,5)$ & $16(23,5)$ & $48(70,6)$ & $20(29,4)$ & $4(5,9)$ & $64(94,1)$ & $8(11,8)$ & $60(88,2)$ & $68(37,0)$ \\
\hline $\begin{array}{l}\text { Karydakis } \\
\text { Flap }\end{array}$ & $25(75,8)$ & $8(24,2)$ & $23(69,7)$ & $10(30,3)$ & $2(6,1)$ & $31(93,9)$ & $2(6,1)$ & $31(93,9)$ & $33(17,9)$ \\
\hline Total* & $\begin{array}{c}152 \\
(82,6)\end{array}$ & $32(17,4)$ & $77(41,8)$ & $107(58,2)$ & $21(11,4)$ & $163(88,6)$ & $29(15,8)$ & $155(84,2)$ & $\begin{array}{c}184 \\
(100,0)\end{array}$ \\
\hline$X^{2}$ value & \multicolumn{2}{|c|}{6,334} & \multicolumn{2}{|c|}{74,474} & \multicolumn{2}{|c|}{6,633} & \multicolumn{2}{|c|}{6,335} & \\
\hline$P$ value & \multicolumn{2}{|c|}{0,042} & \multicolumn{2}{|c|}{0,001} & \multicolumn{2}{|c|}{0,036} & \multicolumn{2}{|c|}{0,042} & \\
\hline
\end{tabular}

* row percentage, $* *$ column percentage

had a BMI below $30\left(\mathrm{~kg} / \mathrm{m}^{2}\right)$. When compared in terms of BMI, no significant difference was found between the treatment methods $(p=0.826)$, (Table 1). It was determined that $70.1 \%$ of the patients smoked, and there was no significant difference between the treatment methods in terms of smoking ( $p=0.901$ ), (Table 1). $9.8 \%$ of a total of 184 patients were re-operated due to relapse, and a statistically significant difference was found between the treatment methods in terms of recurrence $(p=0.012)$. $44.4 \%$ of the relapse cases were applied Limberg flap, $38.9 \%$ Karydakis flap, and $16.7 \%$ primary suturing method (Table 1). $9.2 \%$ of the patients had a history of preoperative abscess drainage. However, a significant difference was found between the treatment methods in terms of preoperative abscess drainage status(0.044). Of those patients who underwent preoperative abscess drainage, $64.7 \%$ were applied Limberg flap, $23.5 \%$ primary suturation, $11.8 \%$ Karydakis flap method (Table 1).

When evaluated in terms of length of hospital stay; $82.6 \%$ of the cases had a hospital stay of 2 days or less, $17.4 \%$ of them 3 days or more. A statistically significant difference was found between the treatment methods when compared in terms of duration of hospital stay $(p=0.042)$. The duration of hospitalization is 2 days or less in $90.4 \%$ of thepatients applied primary suturing, in $76.5 \%$ of those applied Limberg flap method, and in $75.8 \%$ of those applied Karydakis flap method (Table 2). Postoperative drains were present in $41.8 \%$ of the patients, and a statistically significant difference was found between the treatment methods in terms of presence of drains $(p=0.001)$. Accordingly, $7.2 \%$ of those who underwent primary suturing, $70.6 \%$ of those who underwent Limberg flap, and $69.7 \%$ of those who had Karydakis surgery had postoperative drain (Table 2). When treatment methods were compared in terms of recurrence, they were found to differ significantly $(p=0.036)$. Recurrence has developed in $11.4 \%$ of all the cases. According to this,recurrence was detected in $18.1 \%$ of the primary suture group, $5.9 \%$ of the Limberg group and $6.1 \%$ of the Karydakis group (Table 2).

Complications developed in $15.8 \%$ of all the cases in terms of postoperative complications. A statistically significant difference was found between the treatment methods in terms of complications ( $p=$ 0.042). Complications developed in $22.9 \%$ of the primary suture group, $11.8 \%$ of the Limberg flap group, and $6.1 \%$ of the Karydakis group (Table 2).

Of all 29 cases where complications developed, $20.7 \%$ developed seroma, $20.7 \%$ developed hematoma, $41.4 \%$ developed wound-site infection, and $17.2 \%$ developed wound dehiscence. $65.5 \%$ of the total complications are in the primary suturation group, $27.6 \%$ in the Limberg group and $6.9 \%$ in the Karydakis group (Table 3).

\section{DISCUSSION}

Pilonidal sinus disease is a chronic disease especially seen in young men and in the intergluteal region involving the natal clefts in the navicular region. The overall incidence is reported to be $26 / 100.000$. The highest incidence reaches between the ages of 15-30 
Table 3: Distribution of various complications according to treatment methods

\begin{tabular}{|l|c|c|c|c|}
\hline \multirow{2}{*}{ Complications } & \multicolumn{3}{|c|}{ Treatment Methods } \\
\cline { 2 - 4 } & Primary closure & Limberg Flap & Karydakis Flap & Total** \\
\cline { 2 - 4 } & $\mathbf{n ~ ( \% )}$ & $\mathbf{n ~ ( \% )}$ & $\mathbf{n}(\%)$ & $\mathbf{n}(\%)$ \\
\hline Seroma & $3(50,0)$ & $2(33,3)$ & $0(0,0)$ & $16,7)$ \\
\hline Hematoma & $4(66,7)$ & $2(33,3)$ & $1(8,3)$ & $12(41,4)$ \\
\hline Wound-site infection & $8(66,7)$ & $3(25,0)$ & $0(0,0)$ & $2(17,2)$ \\
\hline Wound dehiscence & $4(80,0)$ & $1(20,0)$ & $2(6,9)$ & $29(100,0)$ \\
\hline
\end{tabular}

* row percentage, $* *$ column percentage

and it is seen 3-4 times more in men than in women (6-8). In our study, the mean age was $26.47 \pm 6.10$ and $84.8 \%$ of the cases were male.

Although the etiology of the disease is not fully understood, the main known risk factors include obesity (BMl> $\left.25 \mathrm{~kg} / \mathrm{m}^{2}\right)$, prolonged sitting, deep birth clefts in the navicular region, local trauma, hormonal disorders, poor hygienic behavior, hairy body type and smoking (9-11). When evaluated in terms of BMI, no statistically significant difference was found between the treatment methods in the study. In a study in which four surgical techniques were compared, no difference was found in terms of BMI (12). $70.1 \%$ of the patients in our study were smoking. However, no significant difference was found between the treatment methods in terms of smoking. In most studies comparing treatment methods based on state of smoking, no statistically significant difference was found (13-15).

In our study, $18(9.8 \%)$ of 184 patients were operated as relapse cases. A significant difference was found in terms of treatment method in the relapse cases, and Limberg was applied to $44.4 \%$ of the relapse cases and Karydakis flap method to the $38.9 \%$ of them, which is more than the number of cases that were applied primary suturing. In a study of 303 patients in which four surgical techniques were compared, $10.9 \%$ of the operated cases were relapse cases, and $33.3 \%$ of these cases were applied primary closure, $21.2 \%$ were applied open method, $27.7 \%$ Limberg flap method and $18.1 \%$ Karydakis flap method (12). In another study, $16.2 \%$ of all the operated cases were relapse cases, and the method of leaving open was applied to more than half of these cases (13).

In the study, $9.2 \%$ of the patients had a history of preoperative abscess drainage, and a significant difference was found between the treatment methods in terms of preoperative abscess drainage. Limberg flap technique was applied to $64.7 \%$ of the patients who underwent preoperative abscess drainage. In a study in which preoperative abscess drainage was at $7.1 \%$, contrary to our study, no significant difference was found between the treatment methods in terms of preoperative abscess drainage status (12). In another study, the mean preoperative abscess number was $1.48 \pm 3.91(0-20)$ (13).

In our study, a significant difference was found between the treatment methods in terms of duration of hospital stay, and $90.4 \%$ of the patients who underwent primary suturation had a hospital stay of 2 days and less. In this context, it can be said that the duration of hospital stay is shorter in the patients who underwent primary suturing compared to those who were applied Limberg and Karydakis flap methods. Contrary to most studies in the literature, where no difference was found between the treatment methods in terms of length of hospital stay, there are also other studies showing that the duration of hospital stay is shorter in patients who underwent primary suturing, as in our study $(12,13,16,17,18)$.

In the study, $41.8 \%$ of the patients had postoperative drains, and those with Limberg and Karydakis flaps had approximately 10 times more postoperative drains than in primary suturing. In a study where Ekici et al. compared the treatment methods, the presence of drains was reported in $54.1 \%$ of the patients.

Recurrence developed in $11.4 \%$ of all the cases in the study. When the treatment methods were compared in terms of recurrence, a statistically significant difference was found, with the highest rate of recurrence occurring in the primary suturing group with a rate of $18.1 \%$. Recurrence was detected in $5.9 \%$ of the Limberg group and $6.1 \%$ of the Karydakis group. In studies comparing surgical methods in the literature, the highest recurrence was found in primary suturing (19-22). It has been reported that the recurrence rate in the primary closure method ranges between 4 and $28 \%(23,24)$. In a study in which primary closure and Limberg flaps were compared, recurrence rate was reported as $8 \%$ in patients who underwent Limberg flap method (22). 
In some studies, primary midline closure is seen as a method that should be abandoned due to high recurrence rate and increasing complications (25). In studies, wound site infection, wound dehiscence and seroma rates were reported as 9-20\%, 2.5-16.9\% and $0-2 \%$, respectively $(17,26,27)$. In our study, postoperative complications developed in $15.8 \%$ of all the cases herein. A statistically significant difference was found between the treatment methods in terms of complication status, and complications developed in $22.9 \%$ of the primary suture group, $11.8 \%$ of the Limberg flap group, and $6.1 \%$ of the Karydakis group. The complications that developed in a total of 29 patients were seroma in $20.7 \%$ of the cases, hematoma in $20.7 \%$, wound site infection in $41.4 \%$ and wound dehiscence in $17.2 \% .65 .5 \%$ of the total complications are in the primary suturation group, $27.6 \%$ in the Limberg and $6.9 \%$ in the Karydakis group.

\section{Study limitations}

Due to the retrospective nature of the study, the results of the study are limited to the data in the patients files. Therefore, it is necessary to be careful in generalizing the results to the population.

\section{CONCLUSION}

In conclusion, although primary suturation has a short hospital stay, the rate of recurrence and complications is higher than the Limberg and Karydakis flap methods. In this context, it can be argued that the Limberg and Karydakis flap methods are safer methods than the primary closure method.

Acknowledgement: We thank Associate Professor Binali Catak for helping the statistical analyses.

Funding: This research did not receive any specific grant from funding agencies in the public, commercial, or not-for-profit sectors.

Conflict of interest: All authors declare that they have no conflict of interest.

Peer-review: Externally peer-reviewed.

\section{REFERENCES}

1. lesalnieks I, Ommer A, Petersen S, Doll D, Herold A. German national guideline on the management of pilonidal disease. Langenbecks Arch Surg. 2016; 401: 599-609.

2. Strong E, Callaghan T, Beal E, et al. Patient decision-making and regret in pilonidal sinus surgery: a mixed-methods study. Colorectal Disease. 2021; 00: 1-12.
3. Kallis MP, Maloney C, Lipskar AM. Management of pilonidal disease. Curr Opin Pediatr. 2018; 30: 411-416.

4. Johnson EK. Expert Commentary on Pilonidal Disease: Management and Definitive Treatment. Dis Colon Rectum. 2018; 61: 777-779.

5. Emile SH, Khan SM, Barsom SH, Wexner SD. Karydakis procedure versus Limberg flap for treatment of pilonidal sinus: an updated metaanalysis of randomized controlled trials. International Journal of Colorectal Disease 2021 https://doi.org/10.1007/s00384-021-03922-w.

6. Çiftci F, Abdurrahman I. A different disease: extra sacrococcygeal pilonidal sinuses etiopathogenesis. Int J Clin Exp Med. 2015; 8: 11567-11571.

7. Duman K, Gırgın M, Harlak A. Prevalence of sacrococcygeal pilonidal di-sease in Turkey. Asian J Surg. 2017; 40: 434-437.

8. Gordon KJ, Hunt TM. Osteomyelitis as a complication of a pilonidal sinus. Int J Colorectal Dis. 2016; 31: 155-156.

9. Payne CJ, Walker TWM, Karcher AM, Kingsmore DB, Byrne DS. Are routine microbiological investigations indicated in the manage-ment of non-perianal cutaneous abscesses? Surgeon. 2008 ; 6: 204-206.

10. Bolandparvaz S, Moghadam DP, Salahi R, et al. Evaluation of the risk factors of pilonidalsinus: a sing-le center experience. Turk J Gastroenterol. 2012; 23: 535-537.

11. Doll D, Brengelmann I, Schober P, Ommer A, Bosche $F$, Papalois $A E$ et al. Rethinking the causes of pilonidal sinus disease: a matched cohort study. Scientifc Reports. 2021; 11: 6210

12. Ekici U, Kanlioz M, Ferhatoglu MF, Kartal A. A comparative analysis of four different surgical methods for treatment of sacrococcygeal pilonidal sinus. Asian Journal of Surgery. 2019; 42: 907-913.

13. Keshvari A, Keramati MR, Fazeli MS, Kazemeini A, Nouritaromlou MK. Risk factors for complications and recurrence after the Karydakis flap. Journal of Surgical Research. 2016; 204: 5560.

14. Calis H, Guler Y, Sengul S, Karabulut Z. The effects of perioperative antibiotherapy on surgical site infections in sacrococcygeal pilonidal sinus treated with rhomboid excision and Limberg transposition procedure. Int Wound J. 2019; 16 : 974-978. 
15. Demiryas S, Donmez T. Could Early Postoperative Complications be considered as risk factor for recurrence after pilonidal sinus surgery? Chirurgia. 2019; 114: 475-486.

16. Kartal A, Aydin $\mathrm{HO}$, Oduncu M, Ferhatoğlu MF, Kivilcim T, Filiz Al. Comparison of three surgical techniques in pilonidal sinus surgery. Prague Medical Report. 2018; 119(4): 148-155.

17. Sequeira JB, Coelho A, Marinho AS, Bonet B, Carvalho F, Mor-eira-Pinto J. Endoscopic pilonidal sinus treatment versus total excision with primary closure for sacrococcygeal pilonidal sinus disease in the pediatric population. $J$ Pediatr Surg. 2018; 53(10): 2000-2007.

18. Boshnaq M, Phan YC, Martini I, Harilingam M, Akhtar M, Tsavellas $G$. Limberg flap in management of pilonidal sinus disease: systematic review and a local experience. Acta Chir Belg. 2018; 118(2): 78-84.

19. Luedi MM, Kauf $P$, Evers T, Sievert H, Doll D. Impact of spinal versus general anesthe-sia on postoperative pain and long term recurrence after surgery for pilo-nidal disease. J Clin Anesth. 2016; 33: 236-42

20. Mahdy T. Surgical treatment of the pilonidal disease: Primary closure or flap reconstruction after excision. Dis Colon Rectum. 2008; 51(12): 1816-22.

21. Oueidat D, Rizkallah A, Dirani M, Bou Assi T, Shams A, Jurjus A. 25 years' experience in the management of pilonidal sinus disease. Open Journal of Gastroenterology. 2014; 4: 1-5.

22. Arnous M, Elgendy H, Thabet W, Emile SH, Elbaz SA, Khafagy W. Excision with primary midline closure compared with Limberg flap in the treatment of sacrococcygeal pilo-nidal disease: A randomised clinical trial. Ann $\mathrm{R}$ Coll Surg Engl. 2018; 101: 21-29.

23. Milone $M$, Velotti $N$, Manigrasso $M$, Anoldo $P$, Milone F, DePalma GD. Long-term follow-up for pilonidal sinus surgery: a review of literature with metanalysis. Surgeon. 2018; 16(5): 315-20.

24. Muzi MG, Milito G, Nigro C, Cadeddu F, Farinon AM. A modification of primary closure for the treatment of pilonidal disease in day-care setting. Colorectal Dis. 2009; 11(1): 84-88.

25. Stauffer VK, Luedi MM, Kauf $P$, et al. Common surgical procedures in pilonidal sinus disease: a meta-analysis, merged data analysis, and comprehensive study on recurrence. SciRep. 2018; 8(1): 3058.
26. Kose E, Hasbahceci M, Tonyali H, Karagulle M. Comparative analysis of the same technique-the same surgeon approach in the surgical treatment of pilonidal sinus disease: a retrospective cohort study. Ann Surg Treat Res. 2017; 93(2): 82-87.

27. McCallum IJ, King PM, Bruce J. Healing by primary closure versus open healing after surgery for pilonidal sinus: systematic review and metaanalysis. BMJ. 2008; 336(7649): 868-871. 